Network Working Group

Request for Comments: 2398

FYI : 33

Category: Informational
S. Parker

C. Schmechel

Sun Microsystems, Inc.

August 1998

\title{
Some Testing Tools for TCP Implementors
}

Status of this Memo

This memo provides information for the Internet community. It does not specify an Internet standard of any kind. Distribution of this memo is unlimited.

Copyright Notice

Copyright (C) The Internet Society (1998). All Rights Reserved.

1. Introduction

Available tools for testing TCP implementations are catalogued by this memo. Hopefully disseminating this information will encourage those responsible for building and maintaining TCP to make the best use of available tests. The type of testing the tool provides, the type of tests it is capable of doing, and its availability is enumerated. This document lists only tools which can evaluate one or more TCP implementations, or which can privde some specific results which describe or evaluate the TCP being tested. A number of these tools produce time-sequence plots, see Tim Shepard's thesis [She91] for a general discussion of these plots. Each tools is defined as follows:

Name

The name associated with the testing tool.

Category

One or more categories of tests which the tools are capable of providing. Categories used are: functional correctness, performance, stress. Functional correctness tests how stringent a TCP implementation is to the RFC specifications. Performance tests how 
quickly a TCP implementation can send and receive data, etc. Stress tests how a TCP implementation is effected under high load conditions.

Description

A description of the tools construction, and the implementation methodology of the tests.

Automation

What steps are required to complete the test? What human intervention is required?

Availability

How do you retrieve this tool and get more information about it?

Required Environment

Compilers, OS version, etc. required to build and/or run the associated tool.

References

A list of publications relating to the tool, if any.

2. Tools

2.1. Dbs

Author

Yukio Murayama

Category

Performance / Stress

Description

Dbs is a tool which allows multiple data transfers to be coordinated, and the resulting TCP behavior to be reviewed. Results are presented as ASCII log files.

Automation

Command of execution is driven by a script file. 


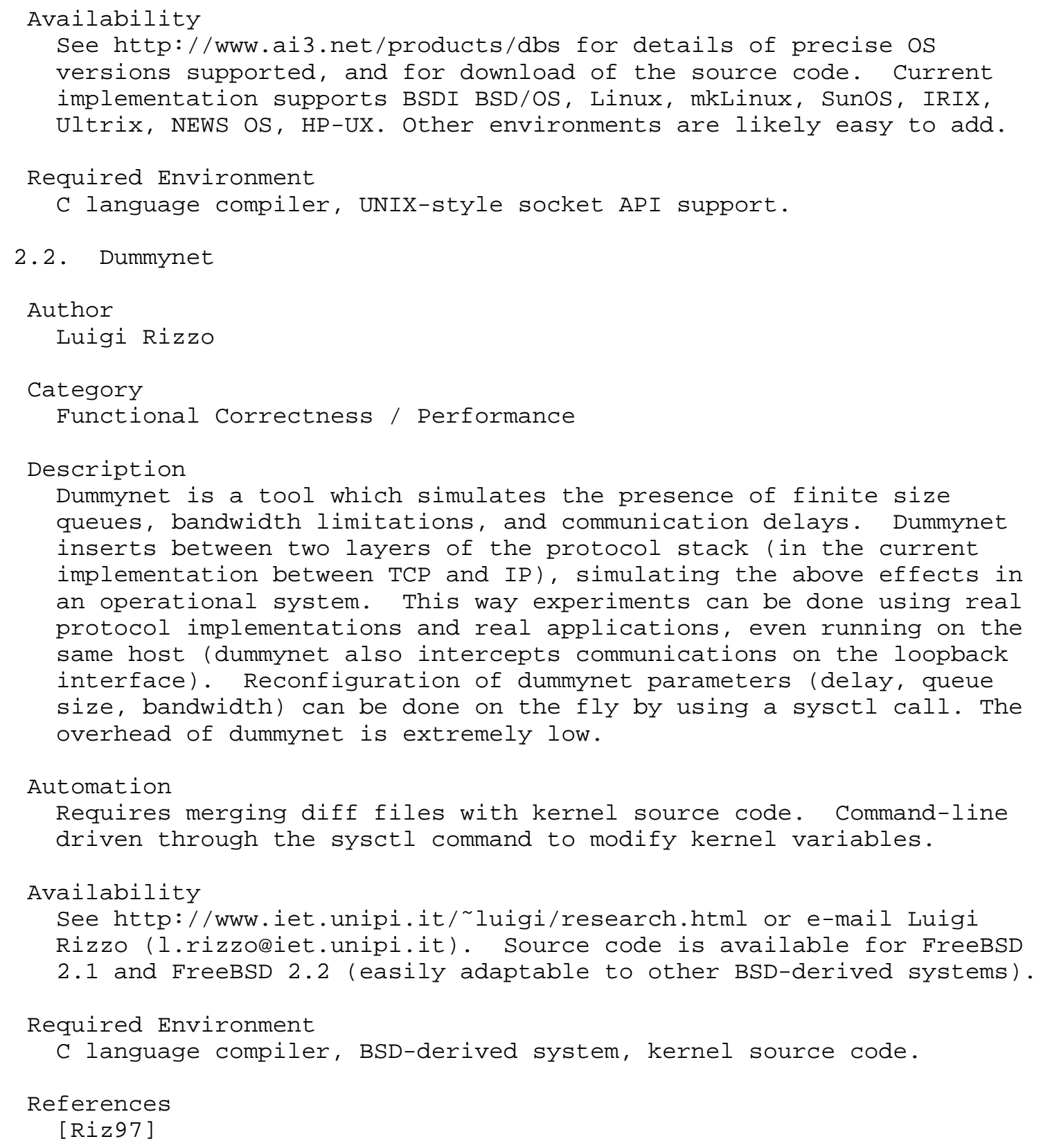




\subsection{Netperf}

Author

Rick Jones

Category

Performance

Description

Single connection bandwidth or latency tests for TCP, UDP, and DLPI. Includes provisions for CPU utilization measurement.

Automation

Requires compilation (K\&R C sufficient for all but-DHISTOGRAM, may require ANSI $C$ in the future) if starting from source. Execution as child of inetd requires editing of /etc/services and /etc/inetd.conf. Scripts are provided for a quick look (snapshot_script), bulk throughput of TCP and UDP, and latency for TCP and UDP. It is command-line driven.

Availability See http://www.cup.hp.com/netperf/NetperfPage.html or e-mail Rick Jones (rajecup.hp.com). Binaries are available here for HP/UX Irix, Solaris, and Win32.

Required Environment

C language compiler, POSIX.1, sockets.

\subsection{NIST Net}

Author

Mark Carson

Category

Functional Correctness / Performance

Description

NIST Net is a network emulator. The tool is packaged as a Linux kernel patch, a kernel module, a set of programming APIs, and command-line and $\mathrm{X}$-based user interfaces.

NIST Net works by turning the system into a "selectively bad" router - incoming packets may be delayed, dropped, duplicated, bandwidthconstrained, etc. Packet delays may be fixed or randomly distributed, with loadable probability distributions. Packet loss may be uniformly distributed (constant loss probability) or congestion-dependent (probability of loss increases with packet queue lengths). Explicit congestion notifications may optionally be sent 
in place of congestion-dependent loss.

Automation

To control the operation of the emulator, there is an interactive user interface, a non-interactive command-line interface, and a set of APIs. Any or all of these may be used in concert. The interactive interface is suitable for simple, spur-of-the-moment testing, while the command-line or APIs may be used to create scripted, non-interactive tests.

Availability

NIST Net is available for public download from the NIST Net web site, http://www.antd.nist.gov/itg/nistnet/. The web site also has installation instructions and documentation.

Required Environment NIST Net requires a Linux installtion, with kernel version 2.0.27 2.0.33. A kernel source tree and build tools are required to build and install the NIST Net components. Building the X interface requires a version of XFree86 (Current Version is 3.3.2). An Athena-replacement widget set such as nextaw (http://www.inf.ufrgs.br/ kojima/nextaw/) is also desirable for an improved user interface.

NIST Net should run on any i386-compatible machine capable of running Linux, with one or more interfaces.

\subsection{Orchestra}

\section{Author}

Scott Dawson, Farnam Jahanian, and Todd Mitton

\section{Category}

Functional Correctness / Performance

\section{Description}

This tool is a library which provides the user with an ability to build a protocol layer capable of performing fault injection on protocols. Several fault injection layers have been built using this library, one of which has been used to test different vendor implementations of TCP. This is accomplished by probing the vendor implementation from one machine containing a protocol stack that has been instrumented with Orchestra. A connection is opened from the vendor TCP implementation to the machine which has been instrumented. Faults may then be injected at the Orchestra side of the connection and the vendor TCP's response may be monitored. The most recent version of Orchestra runs inside the $\mathrm{X}$-kernel protocol stack on the OSF MK operating system. 
When using Orchestra to test a protocol, the fault injection layer is placed below the target protocol in the protocol stack. This can either be done on one machine on the network, if protocol stacks on the other machines cannot be modified (as in the case of testing TCP), or can be done on all machines on the network (as in the case of testing a protocol under development). Once the fault injection layer is in the protocol stack, all messages sent by and destined for the target protocol pass through it on their way to/from the network. The Orchestra fault injection layer can manipulate these messages. In particular, it can drop, delay, re-order, duplicate, or modify messages. It can also introduce new messages into the system if desired.

The actions of the Orchestra fault injection layer on each message are determined by a script, written in Tcl. This script is interpreted by the fault injection layer when the message enters the layer. The script has access to the header information about the message, and can make decisions based on header values. It can also keep information about previous messages, counters, or any other data which the script writer deems useful. Users of Orchestra may also define their own actions to be taken on messages, written in $\mathrm{C}$, that may be called from the fault injection scripts.

Automation

Scripts can be specified either using a graphical user interface which generates Tcl, or by writing Tcl directly. At this time, post-analysis of the results of the test must also be performed by the user. Essentially this consists of looking at a packet trace that Orchestra generates for (in) correct behavior. Must compile and link fault generated layer with the protocol stack.

Availability See http://www.eecs.umich.edu/RTCL/projects/orchestra/ or e-mail Scott Dawson (sdawson@eecs.umich.edu).

Required Environment OSF MK operating system, or X-kernel like network architecture, or adapted to network stack.

References

$[$ DJ94 $],[D J M 96 a],[D J M 96 b]$ 


\subsection{Packet Shell}

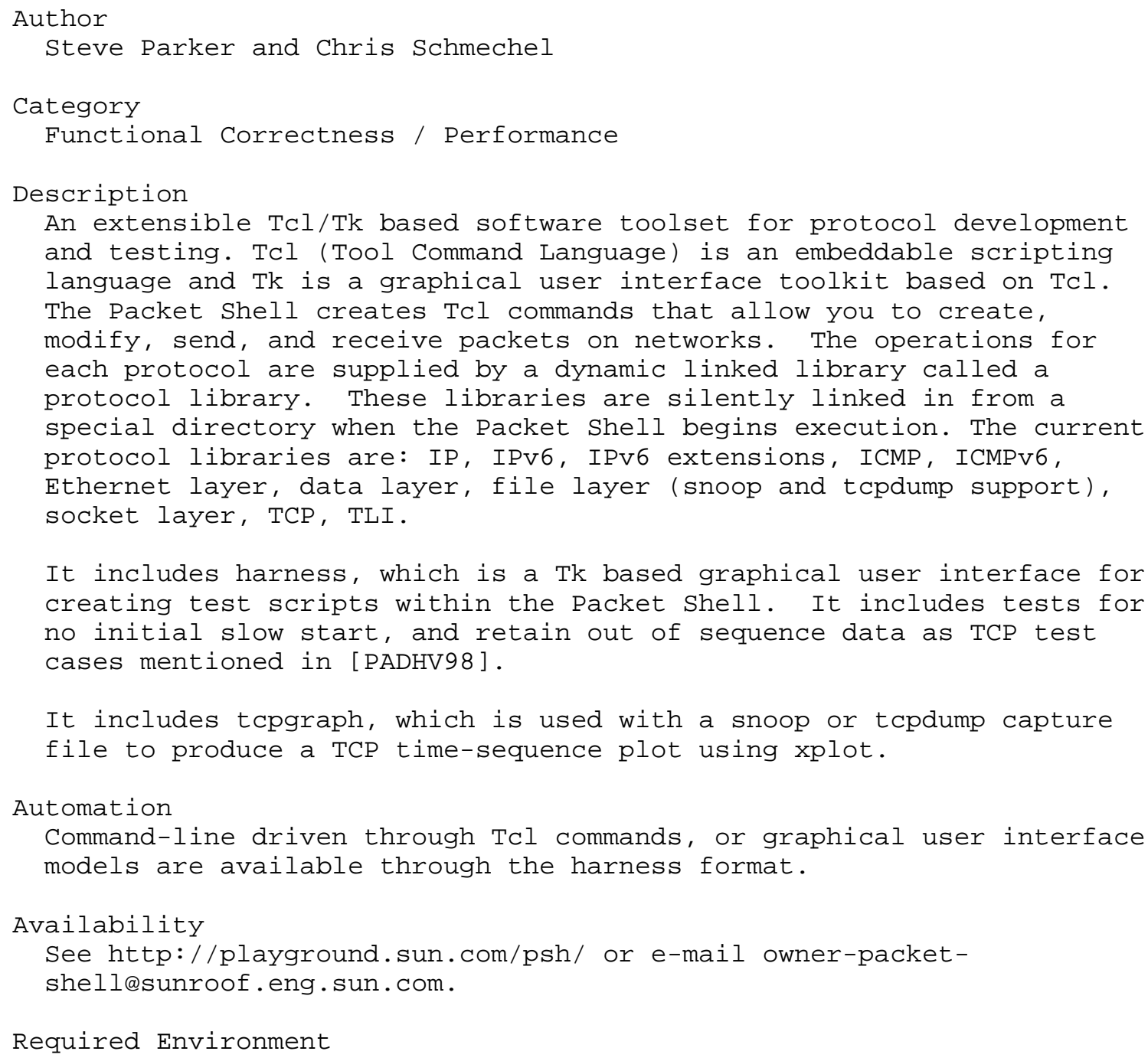




\subsection{Tepanaly}

Author

Vern Paxson

Category

Functional Correctness / Performance

Description

This is a tool for automatically analyzing a TCP implementation's behavior by inspecting packet traces of the TCP's activity. It does so through packet filter traces produced by tcpdump. It has coded within it knowledge of a large number of TCP implementations. Using this, it can determine whether a given trace appears consistent with a given implementation, and, if so, exactly why the TCP chose to transmit each packet at the time it did. If a trace is found inconsistent with a TCP, tcpanaly either diagnoses a likely measurement error present in the trace, or indicates exactly whether the activity in the trace deviates from that of the TCP, which can greatly aid in determining how the traced implementation behaves.

Tcpanaly's category is somewhat difficult to classify, since it attempts to profile the behavior of an implementation, rather than to explicitly test specific correctness or performance issues. However, this profile identifies correctness and performance problems.

Adding new implementations of TCP behavior is possible with tcpanaly through the use of $\mathrm{C}++$ classes.

Automation

Command-line driven and only the traces of the TCP sending and receiving bulk data transfers are needed as input.

Availability Contact Vern Paxson (vern@ee.lbl.gov).

Required Environment C++ compiler.

References

[Pax97a] 


\subsection{Tcptrace}

Author

Shawn Ostermann

Category

Functional Correctness / Performance

Description

This is a TCP trace file analysis tool. It reads output trace files

in the formats of : tcpdump, snoop, etherpeek, and netm.

For each connection, it keeps track of elapsed time, bytes/segments sent and received, retransmissions, round trip times, window

advertisements, throughput, etc from simple to very detailed output.

It can also produce three different types of graphs:

Time Sequence Graph (shows the segments sent and ACKs returned as a function of time)

Instantaneous Throughput (shows the instantaneous, averaged over a few segments, throughput of the connection as a function of time).

Round Trip Times (shows the round trip times for the ACKs as a function of time)

Automation

Command-line driven, and uses the xplot program to view the graphs.

Availability

Source code is available, and Solaris binary along with sample

traces. See http://jarok.cs.ohiou.edu/software/tcptrace/tcptrace.html

or e-mail Shawn Ostermann (ostermanndes.ohiou.edu).

Required Environment

C compiler, Solaris, FreeBSD, NetBSD, HPUX, Linux. 


\subsection{Tracelook}

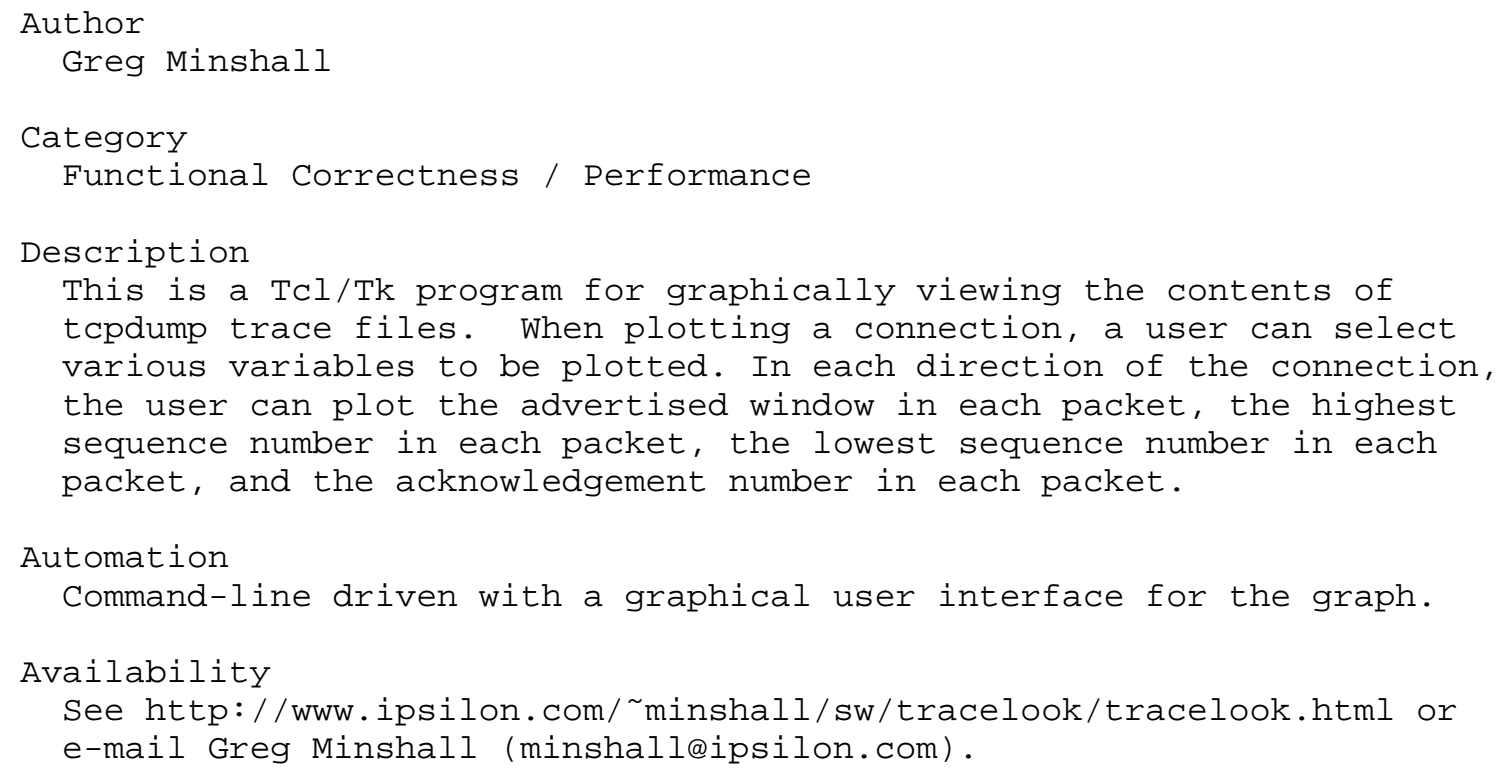




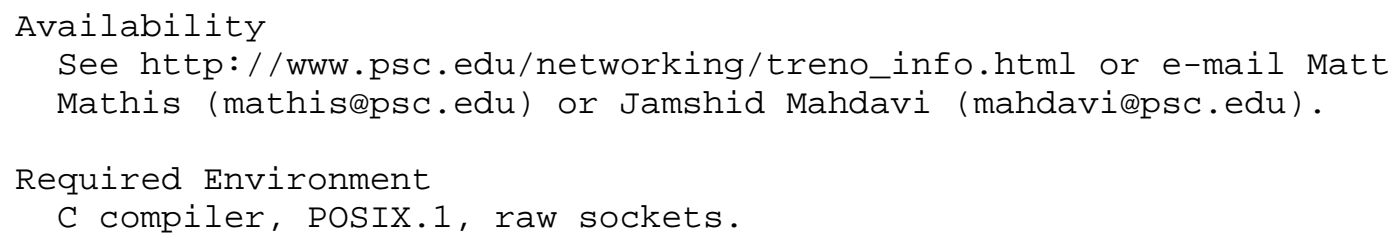




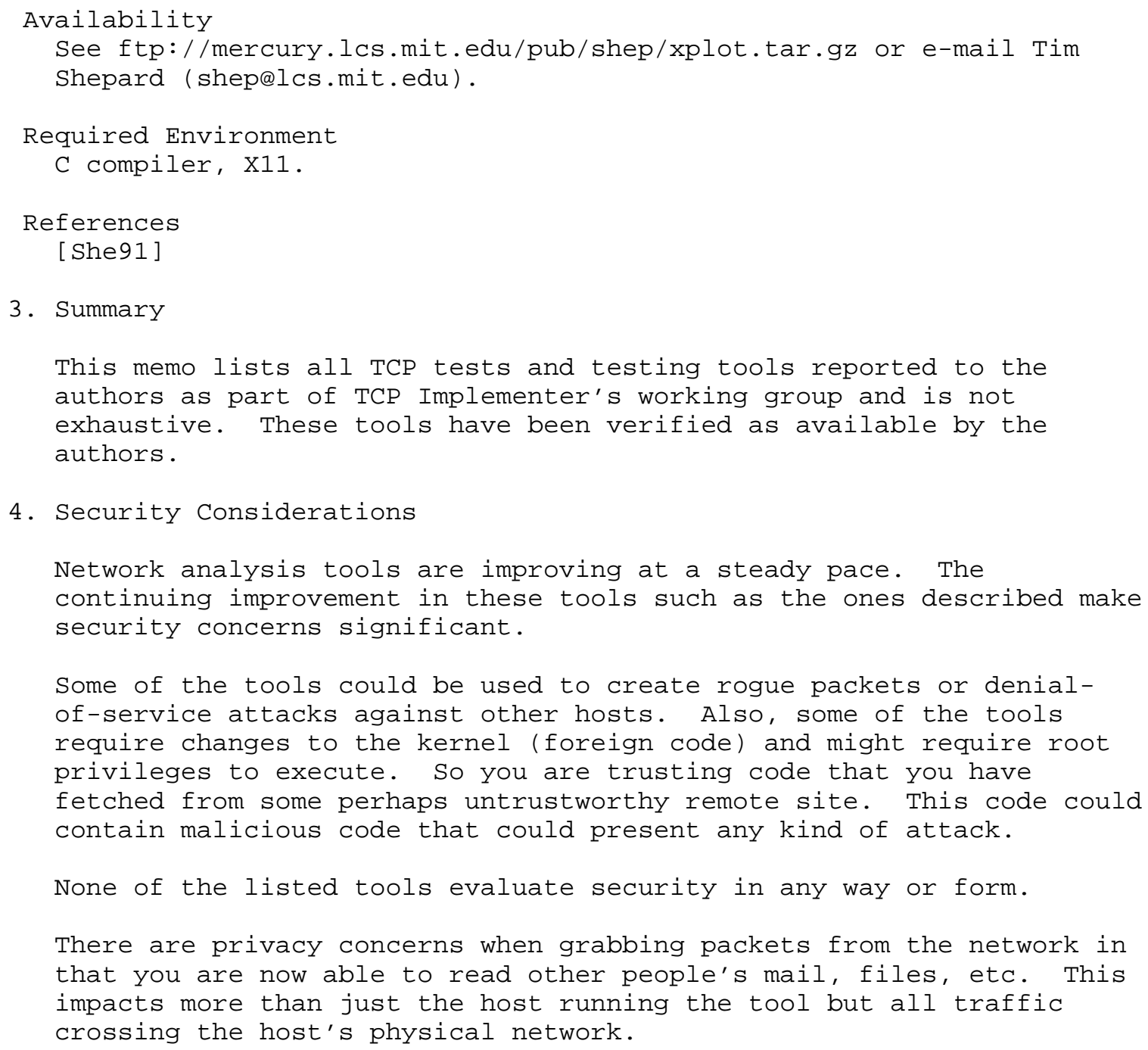

3. Summary

This memo lists all TCP tests and testing tools reported to the authors as part of TCP Implementer's working group and is not exhaustive. These tools have been verified as available by the authors.

4. Security Considerations

Network analysis tools are improving at a steady pace. The continuing improvement in these tools such as the ones described make security concerns significant.

Some of the tools could be used to create rogue packets or denialof-service attacks against other hosts. Also, some of the tools require changes to the kernel (foreign code) and might require root privileges to execute. So you are trusting code that you have fetched from some perhaps untrustworthy remote site. This code could contain malicious code that could present any kind of attack.

None of the listed tools evaluate security in any way or form.

There are privacy concerns when grabbing packets from the network in that you are now able to read other people's mail, files, etc. This impacts more than just the host running the tool but all traffic crossing the host's physical network.

5. References

[DJ94] Scott Dawson and Farnam Jahanian, "Probing and Fault Injection of Distributed Protocol Implementations", University of Michigan Technical Report CSE-TR-217-94, EECS Department.

[DJM96a] Scott Dawson, Farnam Jahanian, and Todd Mitton, "ORCHESTRA: A Fault Injection Environment for Distributed Systems", University of Michigan Technical Report CSE-TR-318-96, EECS Department. 


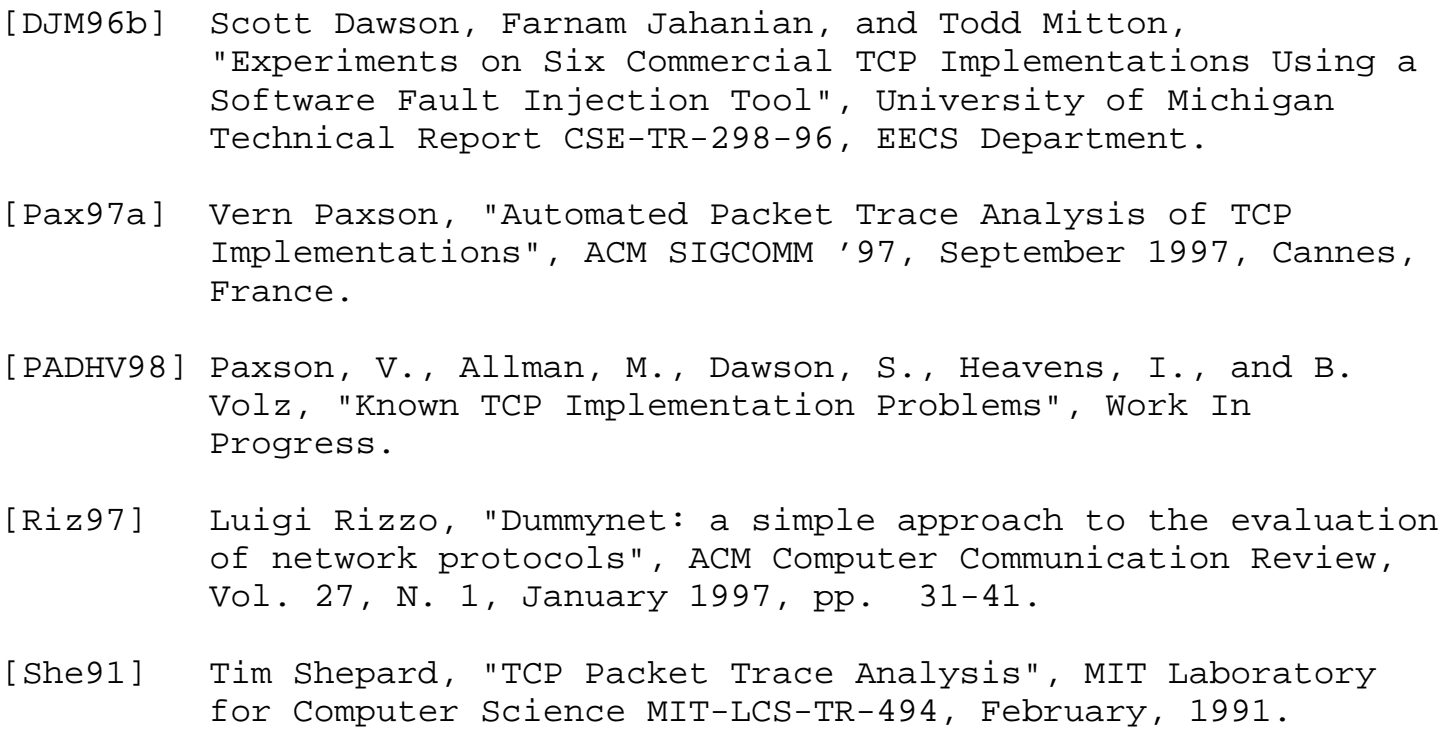

[She91] Tim Shepard, "TCP Packet Trace Analysis", MIT Laboratory for Computer Science MIT-LCS-TR-494, February, 1991. 
6. Authors' Addresses

Steve Parker

Sun Microsystems, Inc.

901 San Antonio Road, UMPK17-202

Palo Alto, CA 94043

USA

Phone: (650) 786-5176

EMail: sparker@eng.sun.com

Chris Schmechel

Sun Microsystems, Inc.

901 San Antonio Road, UMPK17-202

Palo Alto, CA, 94043

USA

Phone: (650) 786-4053

EMail: cschmecleng.sun.com 
7. Full Copyright statement

Copyright (C) The Internet Society (1998). All Rights Reserved.

This document and translations of it may be copied and furnished to others, and derivative works that comment on or otherwise explain it or assist in its implementation may be prepared, copied, published and distributed, in whole or in part, without restriction of any kind, provided that the above copyright notice and this paragraph are included on all such copies and derivative works. However, this document itself may not be modified in any way, such as by removing the copyright notice or references to the Internet society or other Internet organizations, except as needed for the purpose of developing Internet standards in which case the procedures for copyrights defined in the Internet Standards process must be followed, or as required to translate it into languages other than English.

The limited permissions granted above are perpetual and will not be revoked by the Internet society or its successors or assigns.

This document and the information contained herein is provided on an "AS IS" basis and THE INTERNET SOCIETY AND THE INTERNET ENGINEERING TASK FORCE DISCLAIMS ALL WARRANTIES, EXPRESS OR IMPLIED, INCLUDING BUT NOT LIMITED TO ANY WARRANTY THAT THE USE OF THE INFORMATION HEREIN WILL NOT INFRINGE ANY RIGHTS OR ANY IMPLIED WARRANTIES OF MERCHANTABILITY OR FITNESS FOR A PARTICULAR PURPOSE. 